Multidiszciplináris tudományok, 11. kötet. (2021) 5 sz. pp. 353-362 https://doi.org/10.35925/j.multi.2021.5.39

\title{
INTERPOLATED SPLINE METHOD FOR A THERMAL DISTRIBUTION OF A PIPE WITH A TURBULENT HEAT FLOW
}

\author{
Hazim Albedran \\ PhD student, University of Miskolc, Institute of Energy Engineering and Chemical Machinery \\ University of Kufa, Najaf, Iraq \\ 3515 Miskolc, Miskolc-Egyetemváros, e-mail: vegyhnr@uni-miskolc.hu \\ Ali Habeeb Askar \\ PhD student, University of Miskolc, Institute of Physics and Electric Engineering \\ University of Technology - Iraq \\ 3515 Miskolc, Miskolc-Egyetemváros, e-mail: 20156@uotechnology.edu.iq \\ Károly Jármai \\ professor, University of Miskolc, Institute of Energy Engineering and Chemical Machinery \\ 3515 Miskolc,Miskolc-Egyetemváros, e-mail: altjar@uni-miskolc.hu \\ Endre Kovács \\ associate professor, University of Miskolc, Institute of Physics and Electric Engineering \\ 3515 Miskolc, Miskolc-Egyetemváros, e-mail: fizendre@uni-miskolc.hu
}

\begin{abstract}
This work presents an interpolated spline method to mathematically represent experimental data of a thermal distribution on a tube with heat flux. Linear regression was compared with the double linear interpolation process with an optimization algorithm and cubic spline curve method with the proposed problem. The results show that the interpolated experimental data can highly improve the efficiency of the cubic spline curves and lead to a smooth empirical equation for the experiments. The optimization algorithm chooses the interpolated points in a way that provides more minor errors.
\end{abstract}

Keywords: Interpolated spline; Optimization; Curve fitting; Cubic spline; Linear regression; Heat exchanger, Turbulent flow.

\section{Introduction}

Optimization algorithms (Ghafil and Jármai, 2020a) are powerful techniques to find the best possible solution among many other feasible or unfeasible solutions. Artificial bee colony (Ghafil and Jármai, 2018) and particle swarm optimization (Alsamia et al., 2021) are famous examples of metaheuristics (Almufti, 2019) which can be inspired by natural or human-made phneomina (Ghafil et al, 2021). One of the critical applications for optimization is curve fitting (Chen et al., 2005) which is a traditional engineering concept. Many methods were employed to find the best equation representing Cartesian space points like mean square error (Sarbishei and Radecka, 2011), linear regression (Yan and Su, 2003), and splines (De Boor, 1978). Cubic spline curves are piecewise polynomials consisting of adjacent segments. The most crucial engineering application for splines is path planning (Mahmood et al., 2019) in robotics (Ghafil and Jármai, 2019). The heat exchanger (Bouchenna et al., 2021) is a device used to heat transfer between two or more fluids for various applications, including power plants, nuclear reactors, refrigeration and air condition system, automotive industries, heat recovery system, chemical processing and food industries. In order to calculate the heat transfer. Acknowledge the prevailing temperature field 
is the first requirement. Most calculating the temperature distribution methods within a tube wall are given in the literature by constant wall temperature (Askar et al., 2020) or constant heat flux (Karamallah et al., 2016). The heat transfer equipment industry is a vast and expensive part of the world economy due to the cost and size of the heat transfer equipment (Rohsenow et al., 1998). A large part of this cost to the industry is due to rising fuel prices. As a result, methods to enhance the heat transfer process are of great interest. Even small reductions in fuel costs could make the difference between a successful and failing in the power industry (Siddique et al., 2010).

In this paper, interpolated data using a metaheuristic algorithm is used to enhance the efficiency of the cubic spline curve to fit experimental data of a heat exchanger. First of all, the initial control points on the spline are chosen the same as the experimental data; then in-between point is created and chosen by the optimization algorithm. Axiomatically, the position of the control points is affecting the final shape of the spline, so the optimal position of the in-between points is crucial to develop an optimal spline that well-fit data. The interpolated spline method is compared with the pure spline method and linear regression method.

\section{Problem definition}

The physical problem is chosen to be a pipe of $(1.58 \mathrm{~cm})$ outer diameter and $(1.4 \mathrm{~cm})$ inner diameter, with $(150 \mathrm{~cm})$ length as shown in Figure 1 . The outer surface of the tube is electrically heated by a coil made from Nichrome material (Chemical Composition: $80 \% \mathrm{Ni}, 20 \% \mathrm{Cr}$, and has a melting point: $\left.1400^{\circ} \mathrm{C}\right)$ connected to an AC power supply to generate heat flux. It is $(16.7 \mathrm{~mm})$ long and $(1.25 \mathrm{~mm})$ diameter of wire with $(1.36 \Omega / \mathrm{m})$ resistance, and (AWG is 16). An electric insulator of Fiberglass is wrapped around the tube. Drilled ceramic bead elements are inserted around the wire heater to insulate the electrical heater, and then the wire heater is wrapped around the pipe. An Aluminum foil and sectional pipe insulation of glass wool type with $(1.9 \mathrm{~cm})$ internal diameter and $(6.35 \mathrm{~cm})$ outer diameter thermal insulation operating in temperature up to $\left(230^{\circ} \mathrm{C}\right)$ nominal density is $\left(64 \mathrm{~kg} / \mathrm{m}^{3}\right)$ used to insulate the testing tube. The surface temperature distributions along the tube length for different flow rate values are illustrated in Table 1. and Figure 2. The symbol $f$ in Figure 1. and Figure 2. denotes the flow rate in the pipe.

Table. 1. Experimental data of the tube subjected to heat flux.

\begin{tabular}{|c|c|c|c|c|c|c|c|c|c|}
\hline$X(\mathrm{~m})$ & \multicolumn{9}{|c|}{$T\left({ }^{\circ} \mathrm{C}\right)$} \\
\hline 0 & 45.1 & 45.6 & 45.5 & 45.5 & 45.4 & 45.5 & 45.3 & 45.1 & 45.2 \\
\hline 0.22 & 62.1 & 62.3 & 60.1 & 59.5 & 58.9 & 56.3 & 56.2 & 56.1 & 55.2 \\
\hline 0.44 & 65.3 & 63.6 & 61.4 & 61.2 & 61.2 & 57.8 & 57.1 & 56.5 & 55.7 \\
\hline 0.66 & 64.4 & 62.9 & 60.2 & 60.1 & 60 & 57.4 & 56.5 & 55.7 & 55.5 \\
\hline 0.88 & 64.7 & 63.4 & 60.7 & 59.9 & 59.7 & 57.9 & 56.8 & 55.9 & 55.8 \\
\hline 1.1 & 65.6 & 65.1 & 62.1 & 61 & 60.2 & 58.8 & 57 & 56.6 & 56.4 \\
\hline 1.32 & 66.8 & 66.9 & 63.4 & 62.3 & 61.5 & 59.7 & 57.7 & 57.3 & 56.7 \\
\hline 1.54 & 55.3 & 53.5 & 52.2 & 51.3 & 50.6 & 50.2 & 49.9 & 49.6 & 49.4 \\
\hline flow rate (L/min) & 1 & 1.5 & 2 & 2.5 & 3 & 3.5 & 4 & 4.5 & 5 \\
\hline
\end{tabular}




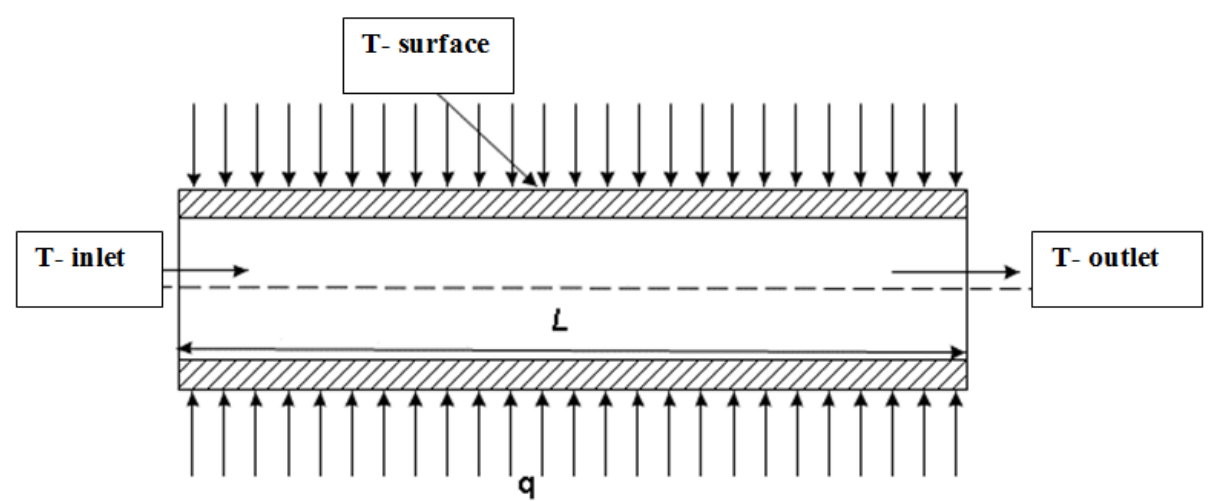

Figure 1. Tube with different flow rates.

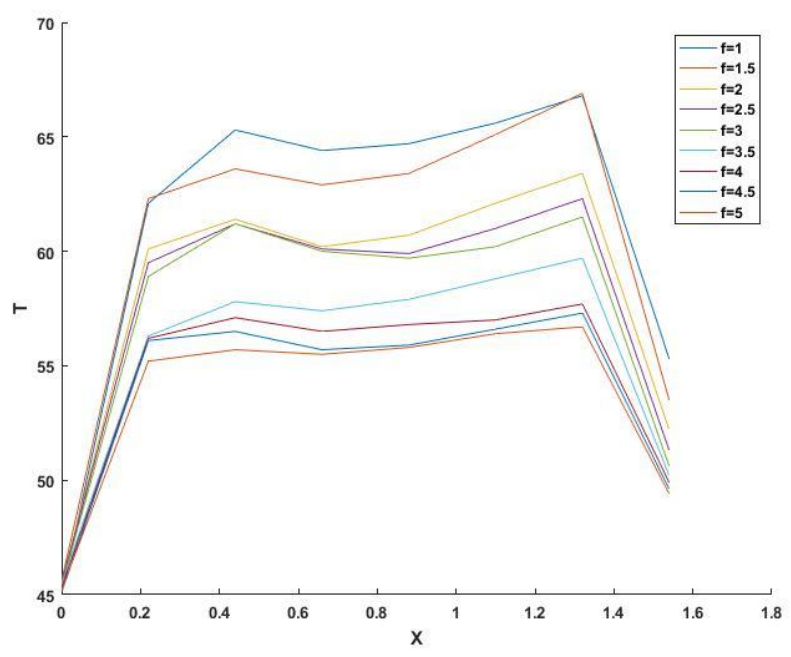

Figure 2. Thermal distribution on the tube with different flow rates.

\section{Polynomial equation}

In this section, a polynomial of the fourth order was proposed to represent the data in section 2, as shown in equation (1). Polynomial regression analysis (Montgomery et al., 2021) was used to find the coefficients of the equation $a_{o}, a_{1}, a_{2}, a_{3}$, and $a_{4}$.

$$
T(x)=a_{o} x^{4}+a_{1} x^{3}+a_{2} x^{2}+a_{3} x+a_{4},
$$

The coefficients in equation (1) are calculated by the polynomial regression method, and they have been found as follows:

$a_{o}=-82.344, a_{1}=264.270, a_{2}=-291.969, a_{3}=130.381, a_{4}=45.048$.

Figure 3., illustrates the real data curve vs polynomial curve denoted as fitting data, for the case flow rate equal to 1 . 


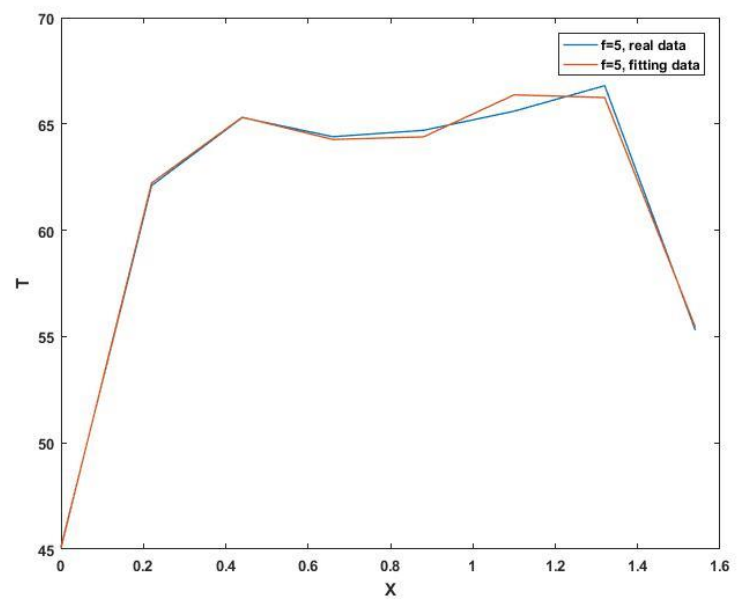

Figure 3. Real data vs polynomial regression curve.

\section{Cubic Spline curves}

Knot spline curve (Likhachev, 2021) is used in this study to represent the experimental data by defining the data set as a control point on the spline. Figure 4. shows the comparison between the knot spline curve and real data from experiments. In this section, knot spline was used purely by defining its control points in terms of real data.

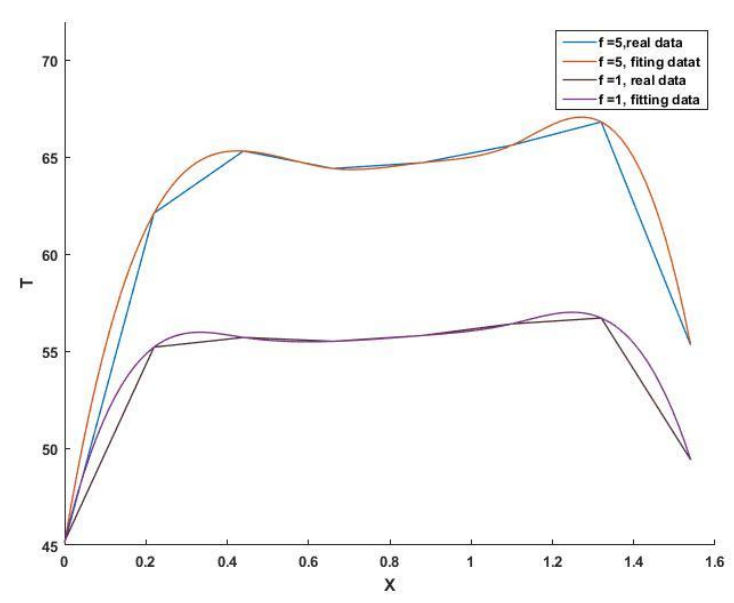

Figure 4. Knot spline curve compared with real data curve.

\section{Interpolated spline curve}

In this section, the Dynamic Differential Annealed Optimization (DDAO) algorithm is presented and used to find the best fitting equation and the results will be compared with other methods. 


\subsection{Dynamic Differential Annealed Optimization}

Dynamic differential annealed optimization is a new metaheuristic that is stimulating the manufacturing process of the dual-phase (DP) steel. Figure 5. reveals the physical scheme behind the algorithm which is used for continuous domains.

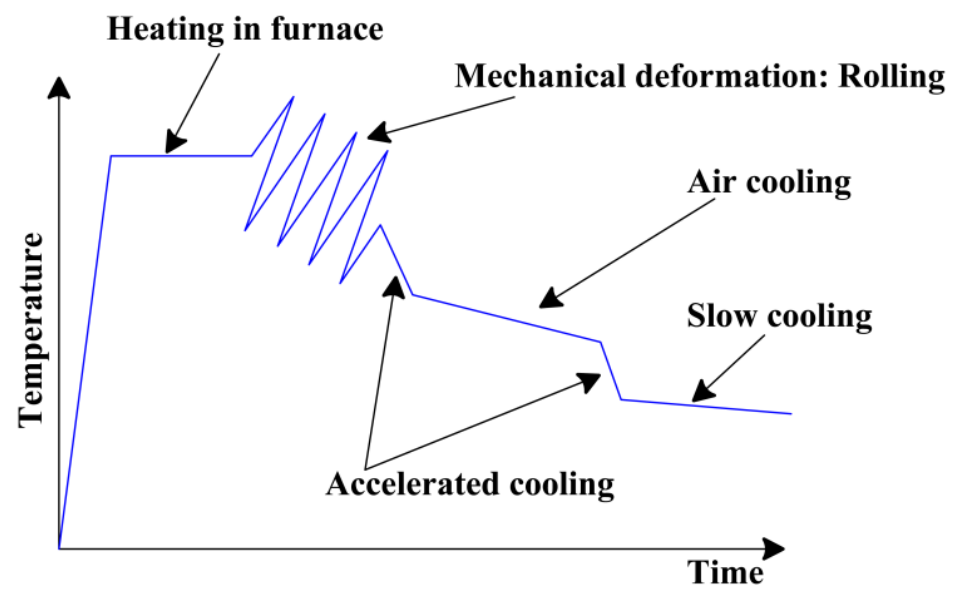

Figure 5. Description of dual-phase steel production process.

DDAO simulates the numerous cooling rates during manufacturing of the DP steel by the following equation:

$$
S_{k}=\left(S c_{i}-S c_{j}\right)+S_{r},
$$

where $S_{k}$ is a new solution proposed for the iteration number $(k), k=1 \ldots n$, where $n$ is the number of iterations, and $S c_{i}$ and $S c_{j}$, are randomly chosen solutions from the population with random $(i)$ and $(j)$ indices. $S_{r}$ is a randomly generated solution within the search space of the problem out of the population. Equation (2) represents the simulation of the mechanical operations during the production

$$
F=\left\{\begin{array}{cl}
1 & \text { if rem }(\text { iteration }, 2)=1 \\
\text { random }[0,1] & \text { if rem }(\text { iteration }, 2)=0
\end{array},\right.
$$

where $F$ is called forging parameter and rem is the remainder after division by 2, and this parameter has two values; 1 at odd iteration numbers, and a random number $[0,1]$ if the iteration number is even. Since forging is done in parallel with cooling, Equation (2) can be modified as follows:

$$
S_{k}=\left(S c_{i}-S c_{j}\right)+S_{r} * F \text {. }
$$

In real production of DP steel, formation of new phases can be occurred at high temperatures more than at low temperatures. To mimic that in mathematics, DDAO has proposed equations (5) and (6):

$$
\begin{gathered}
P=e^{\frac{-\Delta E}{T}}, \\
\Delta E=\frac{\operatorname{Cost}\left(S^{k}\right)-\operatorname{Cost}\left(S_{L}\right)}{\operatorname{Cost}\left(S_{L}\right)},
\end{gathered}
$$


where $P$ is the probability of having a new solution, $\Delta E$ is the difference between the objective value of the proposed solution from equation (4) and the objective value of the solution $S_{L}$, which is a solution of index $L$ in the population, $L=1, \ldots$, population size. $T$ is the temperature variable, which should be damped from a high value to a lower value. Figure 6. shows the pseudocode of the algorithm which is also can be freely downloaded at:

https://www.mathworks.com/matlabcentral/fileexchange/75526-dynamic-differential-annealed-optimization-ddao

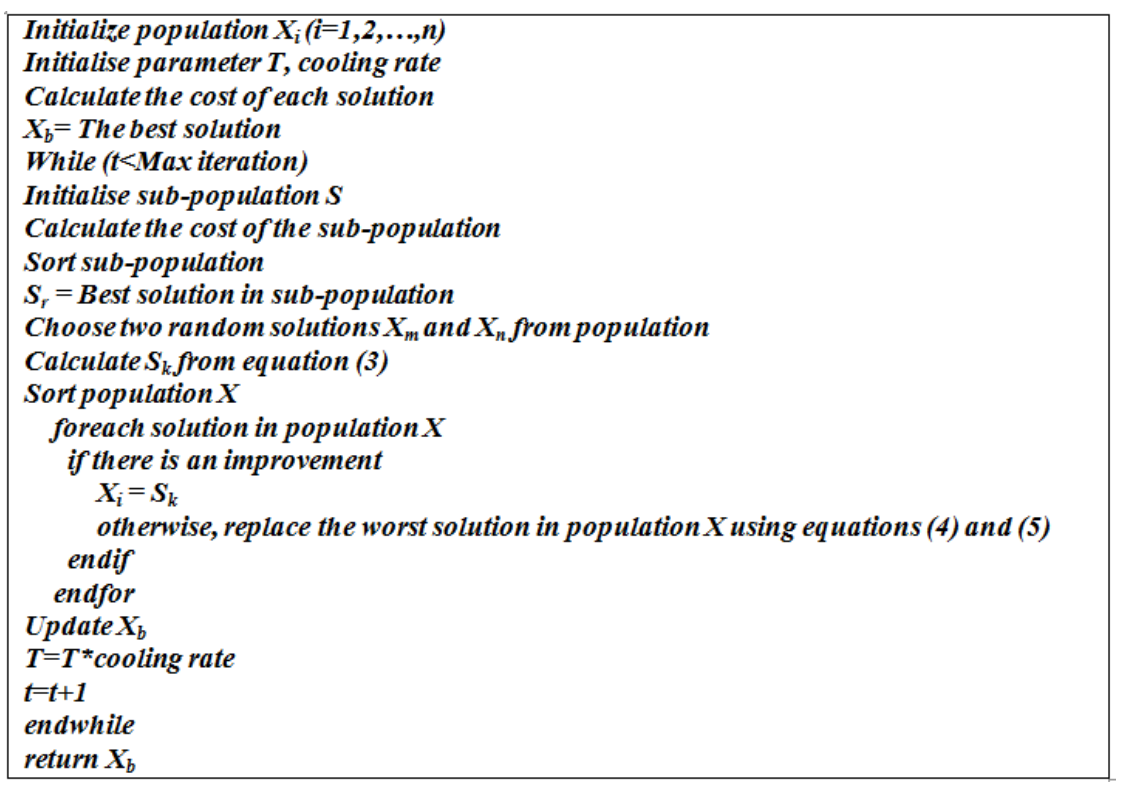

Figure 6. Pseudocode of proposed DDAO algorithm.

\subsection{The objective}

The objective function for the fitting problem can be summrized as the summation of differences among $\mathrm{y}$-axis of the experimental data and the correponding $\mathrm{y}$-axis on the fitting curve on the same $\mathrm{x}$-axis value. The perfect cost function value that can be reached for the fitting problem is zero.

The objective function is described by the following

$$
O=\sum_{i=1}^{n}\left\|y_{p i}-y_{a i}\right\|
$$

where $O$ is the objective function that should be minimized, $y_{p}$ is the y-coordinates on experimental data, $y_{a}$ is the y-coordinates on the fitting curve, and $i$ is the index of the experimental data in the set of $n$ experiments. Figure 7. shows the representation of the objective function which is described by equation (7). 


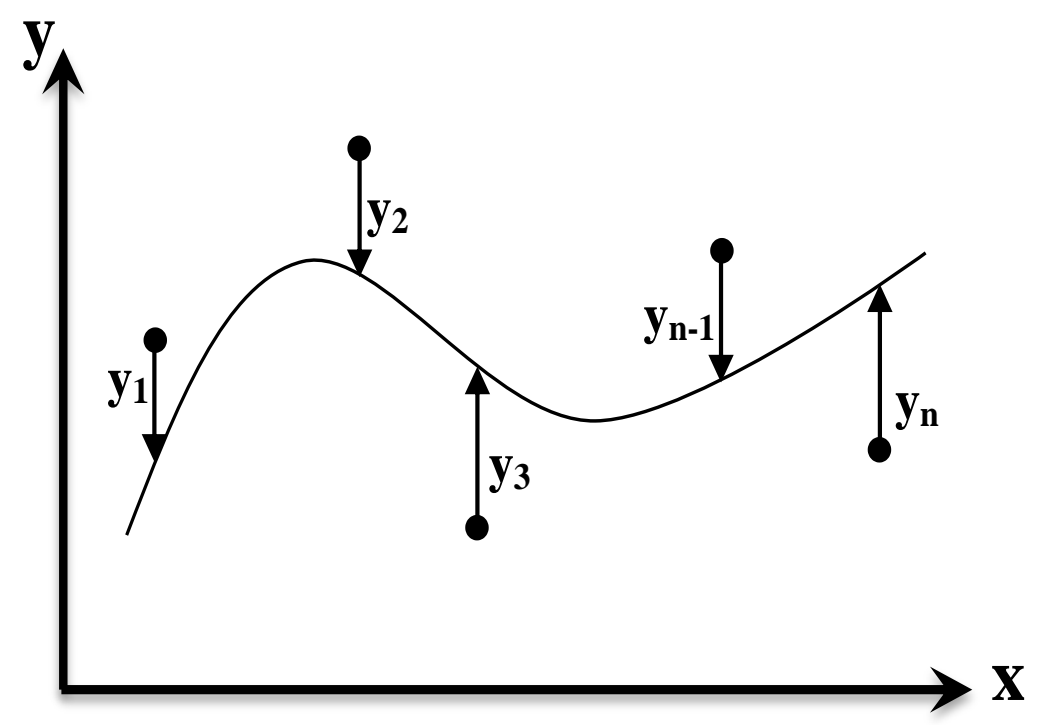

Figure 7. Representation of the objective function.

\subsection{Experiment}

The efficiency of the knot spline curve is improved by considering interpolated points represented by cross symbol in Figure 8. The position of the interpolated points is highly affecting the final shape of the spline, consequently, the efficiency of the fitting process. The domain between adjacent segments is continuous. Therefore, an optimization algorithm is proposed to create and optimize the interpolated points. Dynamic differential annealed optimization (Ghafil and Jármai, 2020b) is used for the optimization problem where it is responsible for the optimal positions of the interpolated points on each successive control point. Figure 9. reveals the interpolated spline curve with experimental data where it is clear that the developed curve greatly matches the experiments.

The cubic spline in Figure 9. representing the experimental data on the continuous domain [0, 1.6] smoothly and perfectly except for a bit of subdomain $[0.23,0.28]$. This is an acceptable error on the method that can be overcome by increasing the number of interpolated points developed by the optimization algorithm. The eight control points which are used in this experiment are the same as the experimental data in Table 1. for a flow rate equal to five. Thus the number of interpolated points are seven considering using one interpolated point between each two successive control points. Each interpolated point should be in the best possible position in order to have the best curve that fits the data. This best position or coordination is estimated by the objective function described in equation (1). The objective function is used by DDAO to return coefficients for equation (1) that are corresponding to the minimum cost function. The output of the DDAO is the red curve in Figure 8. which is consists of 100 points. In other words, We have used the eight control points from experimental data to generate a curve with 100 points using the DDAO with the help of seven interpolated points. The comparison between the performance of the interpolated spline method in Figure 9. and spline method in Figure 10. reveals the efficiency of the proposed method in this article. Table 2. shows the statistical results of comparison pure spline and interpolated spline methods on fitting experimental data in Table 1. 


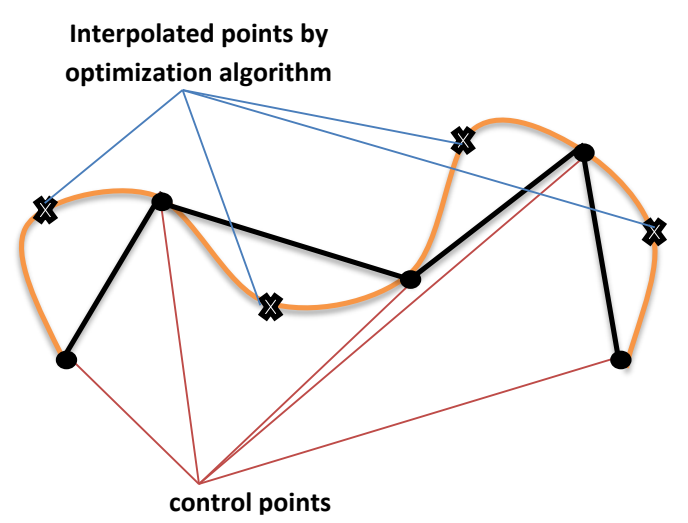

Figure 8. Interpolated spline method.

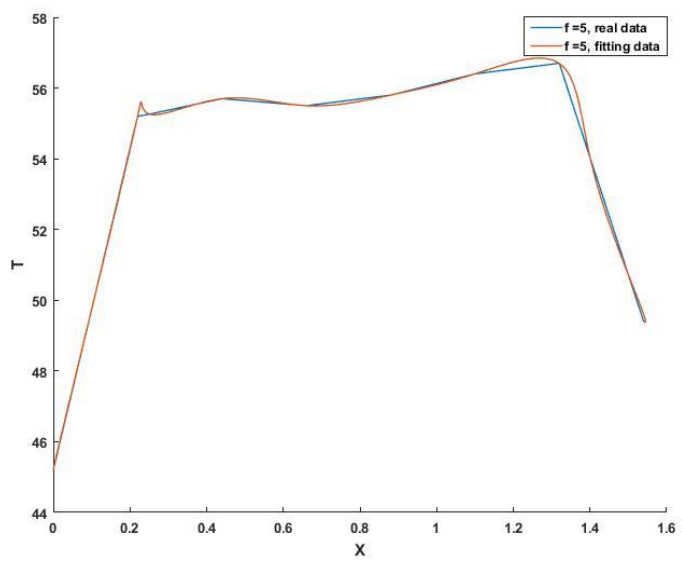

Figure 9. Interpolated spline method compared with experimental data.

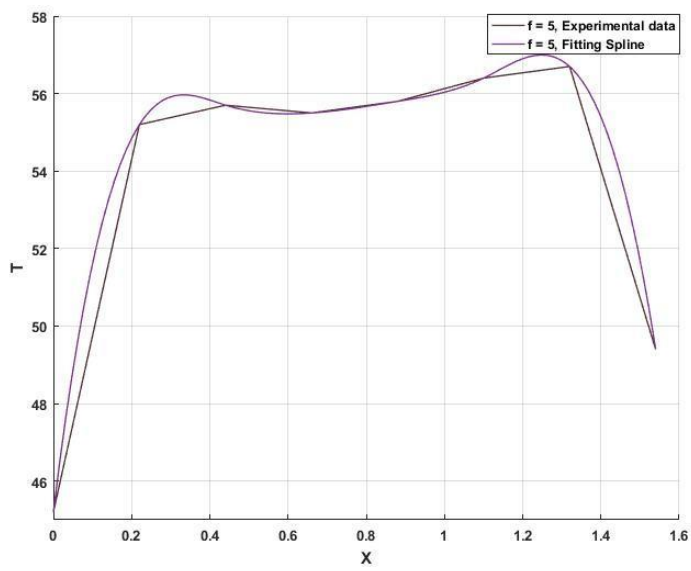

Figure 10. spline method compared with experimental data. 
The error percentage was calculated using the following equation

$$
\text { Error } \%=\frac{\left|y_{f}-y\right|}{y} * 100
$$

where $y$ is the y-coordinate of the experimental data and $y_{f}$ is the y-coordinate of the corresponding $y$ coordinate on the fitting curve. The statistical results reveal that the efficiency of the interpolated spline method is better than spline in fitting experimental data. In many cases, interpolated spline curve is matching the experimental data, and in very narrow ranges there are small overshots that can be an acceptable limitation on the method. The best results were written in bold line in Table 2.

Table. 2. Results of the comparison beween spline and interpolated spline methods.

\begin{tabular}{|l|l|l|l|l|}
\hline \multicolumn{2}{|l|}{ Experimental data } & Method & $\mathrm{y}_{f}$-axis & error\% \\
\hline $\mathrm{x}$-axis & $\mathrm{y}$-axis & & & \\
\hline 0.1 & \multirow{2}{*}{49.74} & Interpolated spline & 49.74 & $\mathbf{0 \%}$ \\
\cline { 3 - 5 } & & spline & 55.89 & $12.3 \%$ \\
\hline \multirow{2}{*}{0.3} & \multirow{2}{*}{0.365} & Interpolated spline & 55.3 & $\mathbf{0 . 1 1 \%}$ \\
\cline { 3 - 5 } & & spline & 55.9 & $0.96 \%$ \\
\hline \multirow{2}{*}{0.5} & \multirow{2}{*}{1.6 .645} & Interpolated spline & 55.715 & $\mathbf{0 . 1 2 \%}$ \\
\cline { 3 - 5 } & & spline & 55.558 & $0.15 \%$ \\
\hline \multirow{2}{*}{56.53} & Interpolated spline & 56.73 & $\mathbf{0 . 3 5 \%}$ \\
\cline { 3 - 5 } & & spline & 56.9 & $0.65 \%$ \\
\hline \multirow{2}{*}{54.4} & Interpolated spline & 54.06 & $\mathbf{0 \%}$ \\
\cline { 3 - 5 } & & spline & 55.401 & $2.4 \%$ \\
\hline
\end{tabular}

\section{Conclusion}

In this paper, interpolated cubic spline curve was developed to fit experimental data and represent them in the best possible equation. The interpolated spline was compared with the linear regression method and pure knot spline on the same experiments. The proposed interpolated spline is overcome linear regression and pure knot spline in fitting the data set. However, the efficiency of the proposed method is depending on the degree of the polynomial in the regression and the number of control points in the spline interpolation.

\section{Acknowledgments}

The research was supported by the Hungarian National Research, Development and Innovation Office under the project number K 134358, and by the NTP-SZKOLL-20-0022 identifier "Focus'21-Focus on community by developing digital competencies" project, supported by the Ministry of Human Resources and Human Resources Support Manager."

\section{References}

[1] Ghafil, H. N., Jármai, K. (2020a). Optimization for Robot Modelling with MATLAB. Springer Nature. https://doi.org/10.1007/978-3-030-40410-9 
[2] Ghafil, H. N., Jármai, K.: Comparative study of particle swarm optimization and artificial bee colony algorithms, 2018 Multiscience XXXII. MicroCAD International Multidisciplinary Scientific Conference, Miskolc-Egyetemváros, Hungary, pp. 1-6. https://doi.org/10.26649/musci.2018.030

[3] Alsamia, S., Ibrahim, D. S., Ghafil, H. N. (2021). Optimization of drilling performance using various metaheuristics. Pollack Period. https://doi.org/10.1556/606.2021.00307

[4] Almufti, S. M. (2019). Historical survey on metaheuristics algorithms. Int. J. Sci. World, 7(1), 1. https://doi.org/10.14419/ijsw.v7i1.29497

[5] Ghafil, H. N., Alsamia, S., Jármai, K. (2021). Fertilization optimization algorithm on CEC2015 and large scale problems. Pollack Period. https://doi.org/10.1556/606.2021.00343

[6] Chen, G., Ren, Z., Sun, H. (2005). Curve fitting in least-square method and its realization with Matlab. Ordnance Ind. Autom., 3, 63.

[7] Sarbishei O., Radecka, K.: Analysis of Mean-Square-Error (MSE) for fixed-point FFT units, 2011 IEEE International Symposium of Circuits and Systems (ISCAS), pp. 1732-1735. https://doi.org/10.1109/ISCAS.2011.5937917

[8] Yan, X., Su, X. G. (2003). Linear regression analysis. Theory Comput.

[9] De Boor, C, De Boor, C. (1978). A practical guide to splines, 27, Springer-Verlag New York. https://doi.org/10.1007/978-1-4612-6333-3

[10] Mahmood, B. A. D., Ghafil, H., Jármai, K. (2019). Optimizing heuristic graph formation with application in kinematic synthesis of a robot arm with revolute joints. J. Eng. Appl. Sci., 14(6), 1948-1976. https://doi.org/10.36478/jeasci.2019.1976.1984

[11] Ghafil, H. N., Jármai, K. (2019). Kinematic-based structural optimization of robots. Pollack Period., 14(3), 213-222. https://doi.org/10.1556/606.2019.14.3.20

[12] Bouchenna, C., Huchet, F., Aramiou, C., Hamard, E., Le Guen, L., Paul, J.-M. (2021). Heat exchanger design based on earthen materials. Energy, 227, 120385. https://doi.org/10.1016/j.energy.2021.120385

[13] Askar, A. H., Kadham, S. A., Mshehid, S. H. (2020). The surfactants effect on the heat transfer enhancement and stability of nanofluid at constant wall temperature. Heliyon, 6(7), e04419. https://doi.org/10.1016/j.heliyon.2020.e04419

[14] Karamallah, A. A., Habeeb, L. J., Asker, A. H. (2016). The effect of magnetic field with nanofluid on heat transfer in a horizontal pipe. Al-Khwarizmi Eng. J., 12(3), 99-109.

[15] Rohsenow, W. M., Hartnett, J. P., Cho, Y. I. (1998). Handbook of heat transfer, vol. 3. McGrawHill, New York.

[16] Siddique, M., Khaled, A.-R., Abdulhafiz, N. I., Boukhary, A. Y. (2010). Recent advances in heat transfer enhancements: a review report. Int. J. Chem. Eng., 2010. https://doi.org/10.1155/2010/106461

[17] Montgomery, D. C., Peck, E. A., Vining, G. G. (2021). Introduction to linear regression analysis. John Wiley \& Sons.

[18] Likhachev, D. V. (2021). On the optimization of knot allocation for B-spline parameterization of the dielectric function in spectroscopic ellipsometry data analysis. J. Appl. Phys., 129(3), 34903. https://doi.org/10.1063/5.0035456

[19] Ghafil, H. N., Jármai, K. (2020b). Dynamic differential annealed optimization: New metaheuristic optimization algorithm for engineering applications. Appl. Soft Comput., 106392. https://doi.org/10.1016/j.asoc.2020.106392 